\title{
Continental-scale spatio-temporal distribution of antibiotic resistance genes in coastal waters along coastline of China
}

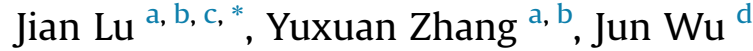 \\ ${ }^{a}$ CAS Key Laboratory of Coastal Environmental Processes and Ecological Remediation, Yantai Institute of Coastal Zone Research (YIC), Chinese Academy of \\ Sciences (CAS), Shandong Key Laboratory of Coastal Environmental Processes, YICCAS, Yantai, Shandong, 264003, PR China \\ ${ }^{\mathrm{b}}$ University of Chinese Academy of Sciences, Beijing, 100049, PR China

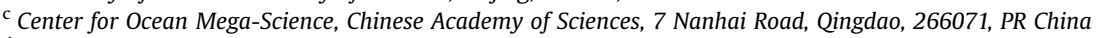 \\ ' School of Resources and Environmental Engineering, Ludong University, Yantai, Shandong, 264025, PR China
}

\section{H I G H L I G H T S}

- Continental-scale pollution of ARGs in coastal waters was firstly investigated.

- Abundances of ARGs in coastal waters ranged from $8.89 \times 10^{1}$ to $4.58 \times 10^{5}$ copies $/ \mathrm{mL}$.

- Mean abundance of ARGs along China's coastline was $8.79 \times 10^{4}$ copies/mL in summer.

- Quinolone resistance genes were the dominant ARGs in coastal waters.

- Tail water and wastewater might be the essential sources of ARGs in coastal waters.

\section{A R T I C L E I N F O}

\section{Article history:}

Received 1 September 2019

Received in revised form

27 October 2019

Accepted 12 January 2020

Available online 13 January 2020

Handling editor: Gang Cao

\section{Keywords:}

Antibiotic resistance genes

Coastal water

Spatio-temporal distribution

Abundance

Continental scale
G R A P H I C A L A B S T R A C T

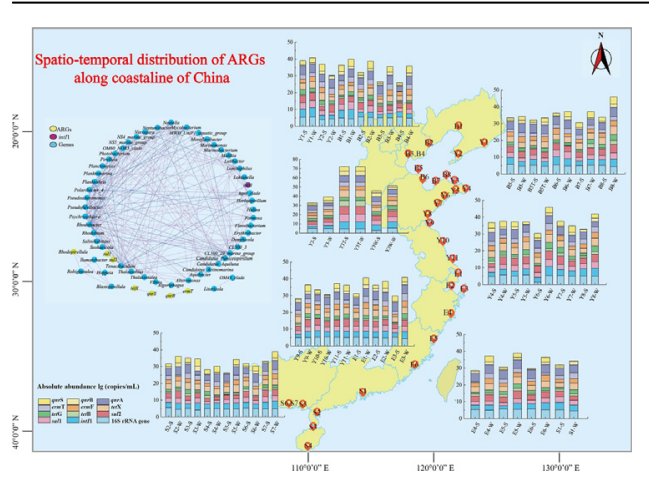

\begin{abstract}
A B S T R A C T
Antibiotic resistance genes (ARGs) have been frequently detected in various matrices all over the world to attract wide attention due to the potential risks. Rare information is available on the pollution of ARGs in the waters of critical ecologically fragile regions such as the coastal zone at a continental scale. Therefore, this study performed field sampling during winter and summer along $18000 \mathrm{~km}$ coastline of China to investigate the distribution of target ARGs in coastal waters at a continental scale. The absolute abundances of ARGs in coastal waters showed drastic spatio-temporal variation with a mean value of $8.79 \times 10^{4} / 1.39 \times 10^{5}$ copies/mL in summer/winter, much lower than those in tail water from the maricultural zone or wastewater. The average absolute abundance of class 1 integron-integrase gene (intI1) in coastal waters was $9.68 \times 10^{3} / 4.15 \times 10^{4}$ copies $/ \mathrm{mL}$ in summer/winter, still lower than that in tail water or wastewater. Quinolone resistance genes were the dominant ARGs in coastal waters to account for over $50 \%$ of total ARGs in most of sampling sites. Bacterial communities in coastal waters showed significant difference both at phylum and genus levels. Abundances of ARGs in coastal waters of this study were comparable with those in other regions previously reported. Tail water and wastewater might be the essential sources of ARGs in coastal waters. The findings of this study provided
\end{abstract}

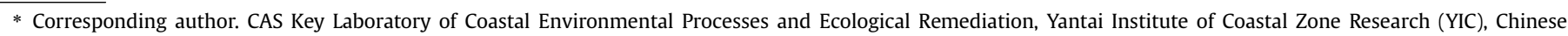
Academy of Sciences (CAS), Shandong Key Laboratory of Coastal Environmental Processes, YICCAS, Yantai, Shandong, 264003, PR China.

E-mail address: jlu@yic.ac.cn (J. Lu).
} 
comprehensive information on the pollution status of ARGs in coastal waters at a continental scale, indicating that ARGs pollution has become a crucial stress affecting the sustainable development of coastal regions.

() 2020 Elsevier Ltd. All rights reserved.

\section{Introduction}

Antibiotic resistance genes (ARGs) have attracted wide attention due to ubiquitous occurrence in different matrices (Yang et al., 2018; Larrañaga et al., 2018; Lv et al., 2018; McCann et al., 2019; Su et al., 2014), easy transfer (Olanrewaju et al., 2019; Qiu et al., 2018), persistence (Calero-Caceres and Muniesa, 2016; Jones et al., 2018), and potential known/unknown health risks (Marti et al., 2018; Bondarczuk et al., 2016). Anthropogenic activities such as aquaculture and wastewater discharge will affect distribution of ARGs in waters (Gao et al., 2018; Huang et al., 2017; Wang et al., 2018). Although ARGs can proliferate under the stress of various pollutants such as antibiotics, polycyclic aromatic hydrocarbons (PAHs) and heavy metals (Chen et al., 2017; Gao et al., 2018; Niu et al., 2016; Xu et al., 2017), antibiotics are still regarded to serve as the main inducer for proliferation of ARGs in the environments. Co-occurrence of antibiotics and ARGs has been frequently observed in different environments (Gao et al., 2018; Niu et al., 2016; Zhang et al., 2018). The abundances of ARGs in different matrices have shown the drastic variations (Gao et al., 2018; Lin et al., 2015; Niu et al., 2016; Zhang et al., 2018), suggesting the complicated existence of ARGs in the environments.

Coastal regions in China have shown fast economical development and these areas are also ecologically fragile due to intense anthropogenic disturbance (Lu et al., 2018). Coastal water pollution in China has been frequently reported in recent years (Lu et al., 2018, 2019a; Pan and Wang, 2012). ARGs have been detected in regional coastal waters and sediments with various abundances (Gao et al., 2018; Lin et al., 2015; Niu et al., 2016; Zhang et al., 2018). However, information on continental-scale spatio-temporal distribution of ARGs in coastal waters is rare. In order to obtain comprehensive information on ARGs pollution in coastal zone, it is necessary to investigate distribution feature of ARGs in coastal waters at a continental scale.

This study performed field sampling along $18000 \mathrm{~km}$ coastline of China during summer and winter to discuss the occurrence and distribution of target ARGs in coastal waters. Influences of different factors on distribution of ARGs were also investigated. The final objective of this study is to provide comprehensive information on spatio-temporal distribution of ARGs in coastal waters at a continental scale.

\section{Materials and methods}

\subsection{Sample collection}

Coastal water samples along the coastline in China were collected in summer (from July 17 to September 30 of 2018) and winter (from October 23 to December 31 of 2017). As shown in Fig. 1, 3535 sampling sites with $35 \times 6$ sub-samples were selected to investigate the distribution of and abundances of ARGs. The detailed information of sampling sites and sampling methods referred to Lu et al. (2018). Additional effluent sample of wastewater treatment plant (WWTP) was collected in Site Y3W and served as comparison for Y3 and Y3T (tail water from the maricultural zone).

\subsection{Analysis of water quality and antibiotics}

Before the analysis of basic water quality and the concentration of antibiotics, water samples were filtered through $0.45 \mu \mathrm{m}$ glass fiber membrane (Merck Millipore Ltd, Ireland). Total nitrogen (TN), total phosphorus (TP), ammonia, nitrate, nitrite, reactive phosphate and reactive silicate were measured by a continuous flow analyzer (Seal, Germany). Total organic carbon (TOC) was determined by a total organic carbon analyzer (Shimadu, Japan). Seventeen antibiotics, involved in four classes, were selected and evaluated for water samples collected in winter, including tetracycline (TC), oxytetracycline (OTC), chlortetracycline (CTC), doxycycline (DOC), sulfamonomethoxine (SMM), sulfadiazine (SDZ), sulfamethazine (SMZ), sulfachinoxalin (SCX), sulfadimethoxine (SDM), sulfameter (SM), sulfaclozine (SCZ), sulfamethoxazole (SMX), norfloxacin (NFC), ciprofloxacin (CFC), ofloxacin (OFC), enrofloxacin (EFC) and roxithromycin (RTM). A portable refractometer (Lohand Biological, China) and a pH meter (INESA, China) were used to measure salinity and $\mathrm{pH}$, respectively. The detailed experimental information and instrument referred to Lu et al. (2018).

\subsection{DNA extraction}

Mixed cellulose esters membrane $(0.22 \mu \mathrm{m}$, Millipore $)$ filtered through a volume of $1.0 \mathrm{~L}$ water sub-sample was used for the extraction of total DNA by TIANamp Soil DNA Kit (TIANGEN Biotech, China) according to instructions. The concentration and purity were measured by NanoDrop Lite (Thermo, USA) and $1 \%$ agarose gel electrophoresis.

\subsection{Quantification of target genes}

A total of twelve genes were quantified by real-time PCR system (qPCR, Bio-Rad CFX384 Touch, USA), including tetracycline resistance genes (tet $\mathrm{B}$, tet $\mathrm{G}$, tet $\mathrm{X}$ ), sulfonamide resistance genes (sul1, sul2), quinolone resistance genes (qnrA, qnrB, qnrS), macrolide resistance genes (erm $\mathrm{T}$, erm $\mathrm{F}$ ), class 1 integron-integrase gene (intI1) and 16S rRNA gene. The primers of target genes, reaction systems, amplification procedures and the establishment of calibration standard curves referred to Wang et al. (2019).

\subsection{Illumina MiSeq sequencing}

The extracted DNA was sent to Majorbio (Shanghai, China) for Illumina MiSeq sequencing (Illumina, USA). The V4-V5 hypervariable region of the 16S rRNA gene was amplified by PCR system (ABI, USA) with primers 515F and 907R (5'-GTGCCAGCMGCCGCGG-3' and $5^{\prime}$-CCGTCAATTCMTTTRAGTTT- $3^{\prime}$ ). The PCR amplified reactions were performed according to the standard program of Majorbio, 3 min of pre-denaturation at $95{ }^{\circ} \mathrm{C}, 27$ cycles of denaturation for $30 \mathrm{~s}$ at $95^{\circ} \mathrm{C}$, annealing for $30 \mathrm{~s}$ at $55^{\circ} \mathrm{C}$, and elongation for $45 \mathrm{~s}$ at $72{ }^{\circ} \mathrm{C}$, and a final extension or $10 \mathrm{~min}$ at $72{ }^{\circ} \mathrm{C}$ with $20 \mu \mathrm{L}$ mixture. Purified amplicons were paired-end sequenced (PE300) in Illumina Miseq platform (Illumina, USA) and the raw data was uploaded in the National Center for Biotechnology Information (NCBI) (Accession Number: SRP219489). Raw sequencing data was quality- 
(a)
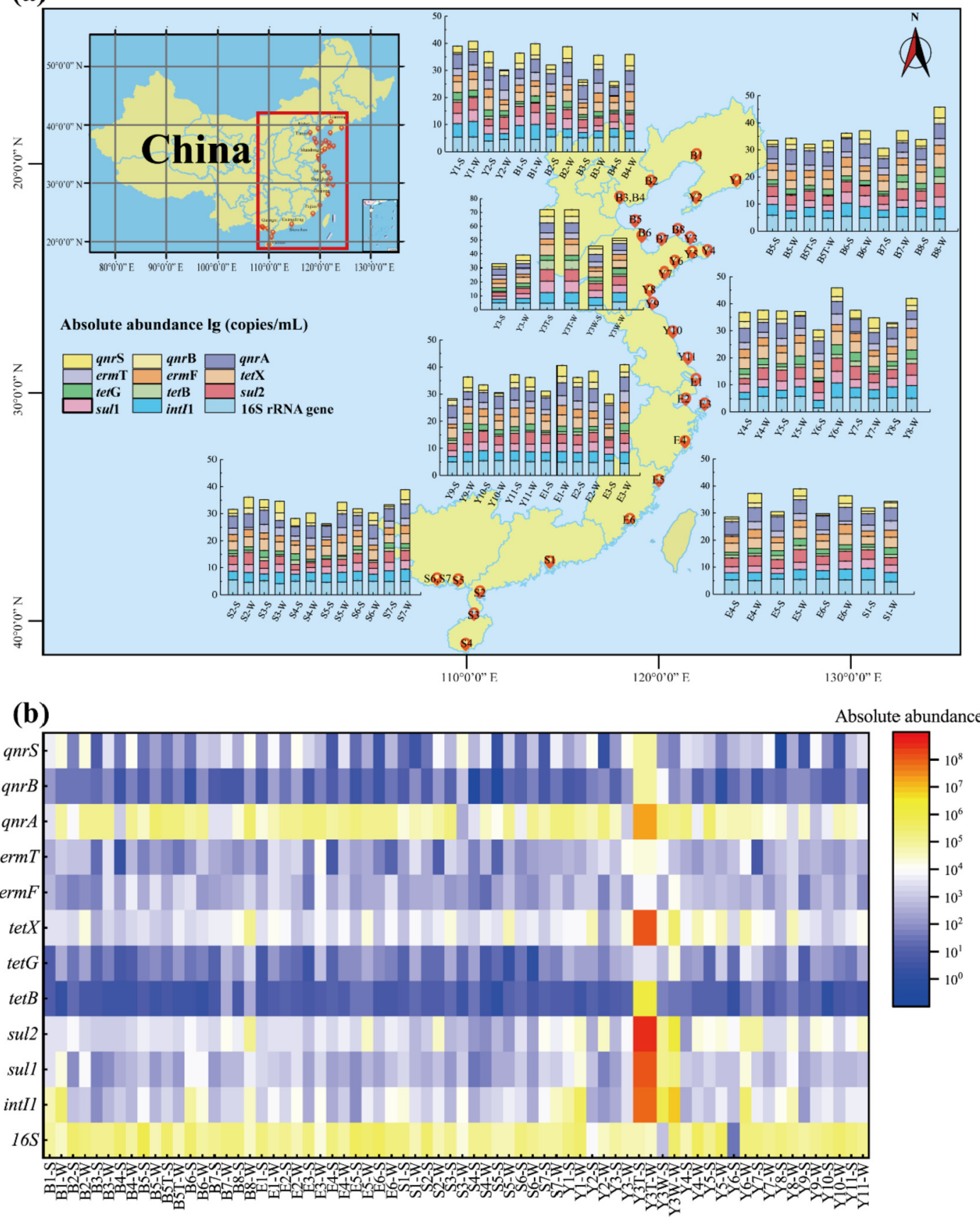

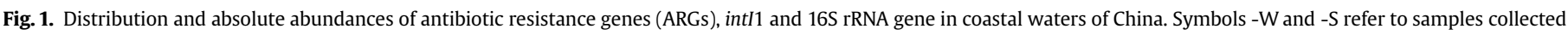
in winter and summer, respectively.

filtered by Trimmomatic and FLASH. UPARSE (version 7.1) was used to cluster the OTUs (operational taxonomic units) with $97 \%$ similarity, and UCHIME was utilized to remove the chimeric sequences. RDP classifier algorithm against the database of Silva was used to classify and analyze the 16S rRNA gene sequences.

\subsection{Statistical analysis}

Pearson and Spearman correlation analysis was accomplished by SPSS 19 (IBM, USA). Principal component analysis (PCA) was conducted by Origin 2019 (Origin Lab Corporation, USA). Cooccurrence network analysis was performed with vegan and igraph packages in $R$ environment, and visualized by Cytoscape 3.7.1. Other data processing and mapping were conducted by Origin 2019.

\section{Results}

3.1. Spatio-temporal distribution of $16 \mathrm{~S}$ rRNA gene, ARGs, and intI1 in coastal waters

The absolute abundances of 16S rRNA gene in coastal waters along the coastline of China showed significant spatio-temporal variations (Fig. 1). Except 3 sites (B5T, Y3T, and Y3W), the 
absolute abundances of $16 S$ rRNA gene in water samples collected from the remaining 32 sites in summer ranged from $2.78 \times 10^{1}$ to $7.43 \times 10^{5}$ copies $/ \mathrm{mL}$ while those in winter varied from $1.68 \times 10^{4}$ to $6.09 \times 10^{5}$ copies $/ \mathrm{mL}$. The lowest absolute abundance of $16 \mathrm{~S}$ rRNA gene in coastal water occurred at site Y6 in summer and site S3 in winter while the highest abundance of 16S rRNA gene in coastal water occurred at site B5 in summer and site Y4 in winter. Except 3 sites (B5T, Y3T, and Y3W), the average absolute abundance of 16S rRNA gene in coastal water of Bohai Area, Yellow Sea Area, East China Sea Area, and South China Sea Area reached $2.72 \times 10^{5}$ / $9.86 \times 10^{4}, 1.30 \times 10^{5} / 2.31 \times 10^{5}, 2.97 \times 10^{5} / 1.19 \times 10^{5}$, and $1.65 \times 10^{5} / 7.01 \times 10^{4}$ copies/mL in summer/winter, respectively. Except 3 sites (B5T, Y3T, and Y3W), the abundances of 16S rRNA gene in coastal water of 11 sites in winter were higher than those in summer. The absolute abundance of 16S rRNA gene in water at Y6 in winter was almost 8000 times that in summer, showing the most significant seasonal variation. The absolute abundances of $16 \mathrm{~S}$ rRNA gene in coastal water at B5T, Y3T, and Y3W were $2.59 \times 10^{5} /$ $5.10 \times 10^{4}, 4.11 \times 10^{4} / 3.89 \times 10^{4}$, and $9.87 \times 10^{2} / 3.23 \times 10^{5}$ copies/ $\mathrm{mL}$ in summer/winter, respectively. The abundances of $16 \mathrm{~S}$ rRNA gene in the mariculture tail water or the effluent of wastewater treatment plants did not show large difference with those in regular coastal water of the study area.

The absolute abundances of ARGs in coastal waters showed drastic spatio-temporal variation (Fig. 1a). In summer, the total absolute abundances of ARGs in coastal waters from 32 sites (except B5T, Y3T, and Y3W) ranged from $3.68 \times 10^{3}$ (B4) to $3.94 \times 10^{5}(\mathrm{Y} 5)$ copies/mL with a mean value of $8.79 \times 10^{4}$ copies/ $\mathrm{mL}$ while those in water from B5T, Y3T, and Y3W varied from $2.44 \times 10^{5}(\mathrm{~B} 5 \mathrm{~T})$ to $6.05 \times 10^{8}(\mathrm{Y3T})$ copies $/ \mathrm{mL}$. In winter, the total absolute abundances of ARGs in coastal waters from 32 sites (except B5T, Y3T, and Y3W) varied from $2.76 \times 10^{4}(\mathrm{~S} 3)$ to $5.41 \times 10^{5}(\mathrm{~B} 8)$ copies/mL with an average value of $1.39 \times 10^{5}$ copies/mL while those in water from B5T, Y3T, and Y3W ranged from $2.12 \times 10^{5}(\mathrm{~B} 5 \mathrm{~T})$ to $6.09 \times 10^{8}(\mathrm{Y3T})$ copies $/ \mathrm{mL}$. The total absolute abundances of ARGs in water of 10 sites (Y1, B5T, Y4, Y5, Y7, Y9, E2, E3, E5, and S3) in summer were higher than those in winter. Interestingly, the total absolute abundance of ARGs in water of 3 sites (B3, Y3T, and E6) in winter were slightly higher than those in summer, suggesting that the temperature did not have significant effects on distribution of ARGs in these sites. Except 3 sites (B5T, Y3T, and Y3W), the average total absolute abundance of ARGs in different areas followed the order of East China Sea Area > Yellow Sea Area $>$ South China Sea Area $>$ Bohai Area in summer and Bohai Area $>$ East China Sea Area $>$ South China Sea Area $>$ Yellow Sea Area in winter. Absolute abundances of ARGs in water at Y3T were generally higher than those of the remaining sites by 3-4 orders of magnitude in both summer and winter, suggesting tail water might be an important source of ARGs. Absolute abundances of ARGs in water at B5T in summer and winter did not show significant difference with those in coastal water of the other sites, suggesting some treatment techniques used in mariculture systems might have positive effects on reducing ARGs in tail water.

Quinolone resistance genes ( $q n r)$ were the dominant ARGs in coastal water (Fig. 1a and b). Absolute abundances of qnr accounted for approximately $57.0 \%-96.8 \%$ of total abundances of ARGs in water samples collected from $71.4 \%$ of sampling sites in winter while those covered about $53.9 \%-98.8 \%$ of total abundances of ARGs in coastal water of 26 sampling sites in summer. Absolute abundances of sulfonamide resistance genes (sul) accounted for approximately $51.3 \%-84.1 \%$ of total abundances of ARGs in water samples collected from 5 sites (B7, Y3T, Y3W, Y9, and Y11) in winter while those covered about $40.0 \%-80.1 \%$ of total abundances of ARGs in water samples collected from 6 sites (B2, B4, Y3T, Y3W, Y7, and Y10). Absolute abundances of tetracycline resistance genes (tet) covered about $30.4 \%-60.3 \%$ of total abundances of ARGs in water samples collected from 6 sites (Y3-Y6, Y8, and S5) in winter while those accounted for approximately $31.8 \%-46.4 \%$ of total abundances of ARGs in water from 4 sites (Y2, B7, B8, and S4). In contrast, absolute abundances of marcrolide resistance genes (erm) accounted for less than $7 \%$ of total abundances of ARGs in water from 34 sites (except Y3) in summer and winter.

The absolute abundances of intI 1 in coastal waters along coastline of China showed magnificent seasonal and temporal variation (Fig. 1a and b). Except 3 sites (B5T, Y3T, and Y3W), the absolute abundances of ARGs in water samples collected from the remaining 32 sites in summer/winter were in the range of $\left(8.89 \times 10^{1}-9.57 \times 10^{4}\right) /\left(1.56 \times 10^{2}-4.58 \times 10^{5}\right)$ copies $/ \mathrm{mL}$ with the mean value of $9.68 \times 10^{3} / 4.15 \times 10^{4}$ copies $/ \mathrm{mL}$. The lowest abundance of intI1 in coastal water occurred at site B3 in summer and Y2 in winter while the highest abundance of intI1 in water occurred at site Y3T both in summer and winter. Except 3 sites (B5T, Y3T, and Y3W), the average absolute abundance of intI1 in coastal water of different areas followed the orders of Bohai Area $>$ Yellow Sea Area $>$ South China Sea Area $>$ East China Sea Area in summer and Yellow Sea Area $>$ Bohai Area $>$ East China Sea Area $>$ South China Sea Area in winter. Gene intI1 in coastal water of about $74 \%$ of sampling sites showed higher absolute abundances in winter. Absolute abundances of intI 1 in water at Y3T and Y3W were generally higher than those of the remaining sites by 2-5 orders of magnitude, showing that tail water and effluent of wastewater treatment plants possessed higher possibility for transfer of ARGs since intI1 was generally an indicator for ARGs transfer (Lu et al., 2019b, 2019c; Wang et al., 2019).

\subsection{Bacterial community of coastal waters}

All samples collected in summer were further measured by high-throughput sequencing for exploring the bacterial communities of coastal waters along coastline of China. Bacterial communities of different water samples showed significant difference both at phylum and genus levels (Fig. 2). Proteobacteria, Bacteroidetes, Cyanobacteria, and Actinobacteria were 4 dominant phyla in all coastal water samples (Fig. 2a). Percentages of Proteobacteria in total bacterial phyla of water samples ranged from $22.51 \%$ (B5) to $77.77 \%$ (Y4) with an average of $73.05 \%$. Average percentages of Proteobacteria in total bacterial phyla of water samples collected from different areas followed the order of East China Sea Area $>$ Yellow Sea Area $>$ South China Sea Area $>$ Bohai Area. Percentages of Bacteroidetes in total bacterial phyla of water samples ranged from $4.09 \%$ (E3) to $41.04 \%$ (B6) with a mean value of $15.45 \%$. Average percentage of Bacteroidetes in total bacterial phyla of water samples collected from East China Sea Area was the lowest while that of water samples collected from Bohai Area reached the highest. Percentages of Cyanobacteria in total bacterial phyla of water samples ranged from $1.15 \%$ (Y4) to $51.07 \%$ (B5). Percentages of Actinobacteria in total bacterial phyla of water samples ranged from $0.76 \%(\mathrm{Y} 2)$ to $34.00 \%$ (Y1) with a mean value of $9.30 \%$. Maximal percentage of Planctomycetes and Acidobacteria in total bacterial phyla of water sample E1 reached $8.90 \%$ and $8.02 \%$, respectively.

Bacterial communities of coastal waters showed more complicated composition at genus level (Fig. 2b). Except some unclassified or unranked genera, 3 bacteria including Vibrio, Synechococcus, and Candidatus_Pelagibacter were the dominant genera of the bacterial communities of coastal waters. Vibrio accounted for approximately $4.00 \%$ of total bacterial genera in coastal waters on average with a maximal percentage of $27.43 \%$. Percentages of Vibrio in total bacterial genera of water samples collected from East China Sea Area were significantly higher than those of water samples collected from the remaining areas. Synechococcus covered about $0.00 \%-$ 
(a)

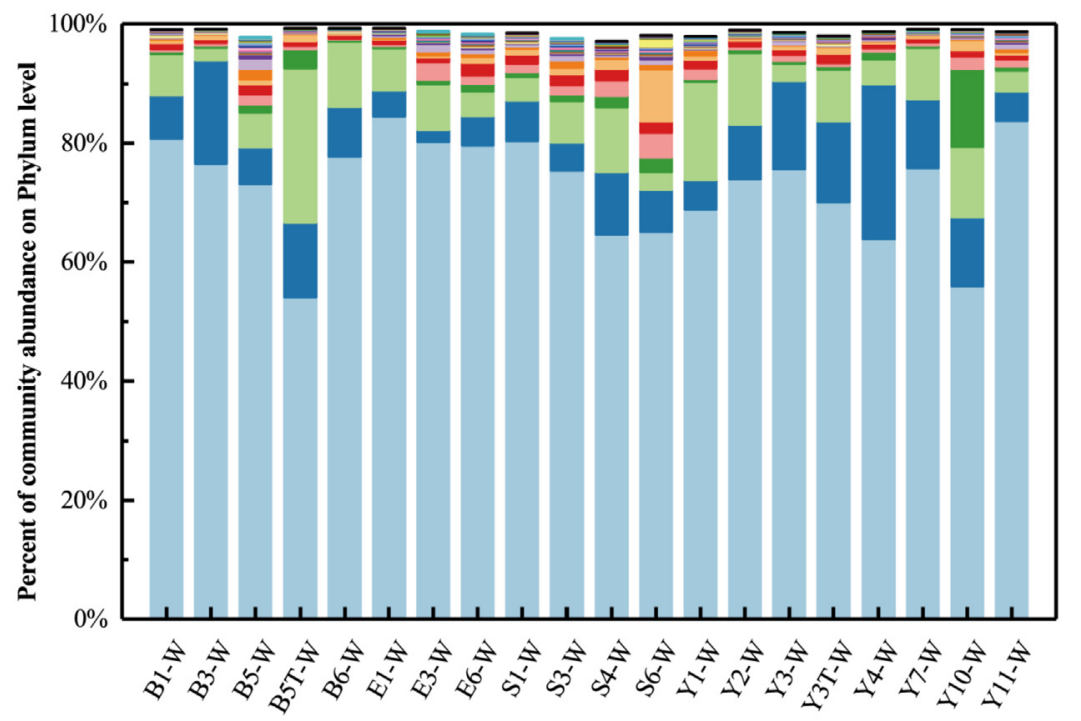

\begin{tabular}{|c|c|c|c|}
\hline Synergistetes & Candidatus_Moranbacteria & Candidate_division_NC10 & Ignavibacteriae \\
\hline Thermotogae & Fusobacteria & Candidate_division_WOR_3 & Candidatus_Nomurabacteria \\
\hline Candidate_division_Zixibacteria & Aquificae & Armatimonadetes & Lentisphaerae \\
\hline Candidatus_Dadabacteria & Candidatus_Saccharibacteria & Tenericutes & Chlamydiae \\
\hline Nitrospinae & Candidatus_Parcubacteria & Chlorobi & Candidatus_Woesebacteria \\
\hline Deinococcus_Thermus & Candidatus_Tectomicrobia & Nitrospirae & Spirochaetes \\
\hline Candidatus_Giovannonibacteria & Acidobacteria & Gemmatimonadetes & Chloroflexi \\
\hline Verrucomicrobia & Firmicutes & Planctomycetes & Cyanobacteria \\
\hline Actinobacteria & Bacteroidetes & Proteobacteria & \\
\hline
\end{tabular}

(b)

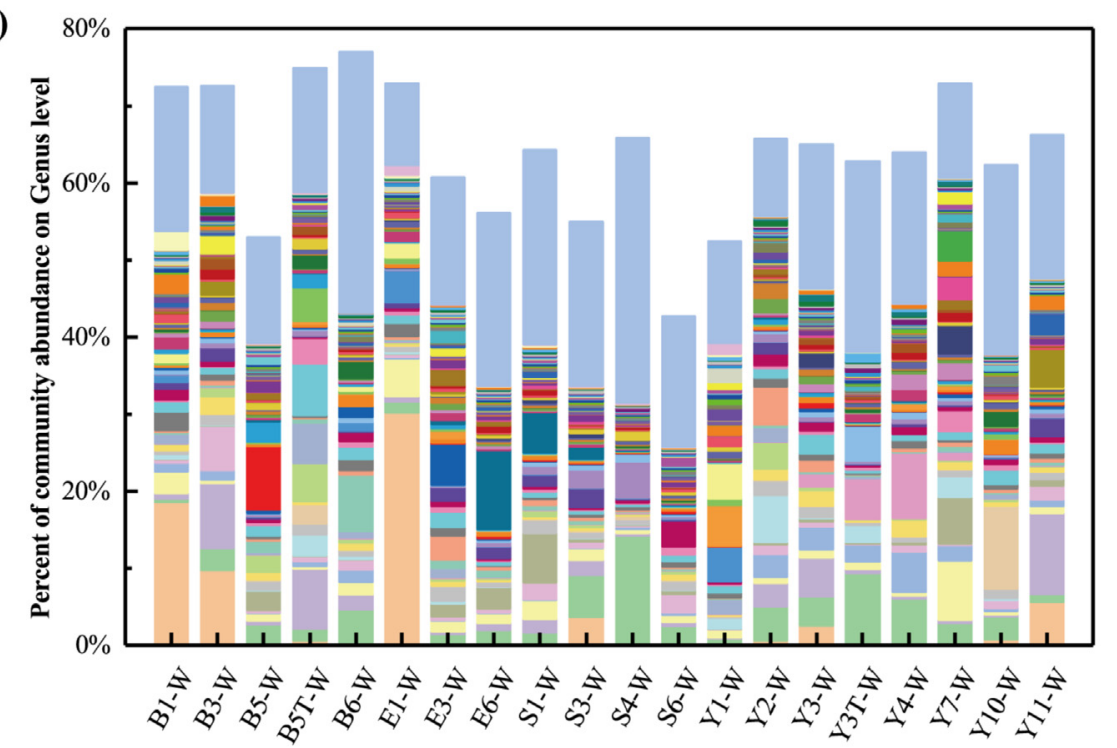

\begin{tabular}{|l|l}
\hline Others \\
\hline Crocinitomix \\
Acidovorax \\
\hline Mesorhizobium \\
\hline Pseudorhodobacter \\
\hline Novosphingobium \\
\hline Fluviicola \\
\hline Flavobacterium \\
\hline Mycobacterium \\
\hline Sphingopyxis \\
\hline Candidatus_Actinomarina \\
\hline Paracoccus \\
\hline Gordonia \\
\hline Ruegeria \\
Pseudomonas
\end{tabular}

\begin{tabular}{|c|c|}
\hline Acidimicrobium & Bacteriovorax \\
\hline Celeribacter & Bradyrhizobium \\
\hline Sphingobium & Sphingomonas \\
\hline Amphritea & Aestuariivita \\
\hline Neptuniibacter & Aliiroseovarius \\
\hline Gemmatimonas & Oceanobacter \\
\hline Rhodobacter & Oceanospirillum \\
\hline Leisingera & Candidatus_Aquiluna \\
\hline Oleiphilus & Pseudooceanicola \\
\hline Oceanicola & Spongiibacter \\
\hline Limnohabitans & Marinobacterium \\
\hline Roseovarius & Loktanella \\
\hline Planktomarina & Alteromonas \\
\hline Rhodococcus & Alcanivorax \\
\hline Vibrio & Candidatus_Pelagibacte \\
\hline
\end{tabular}
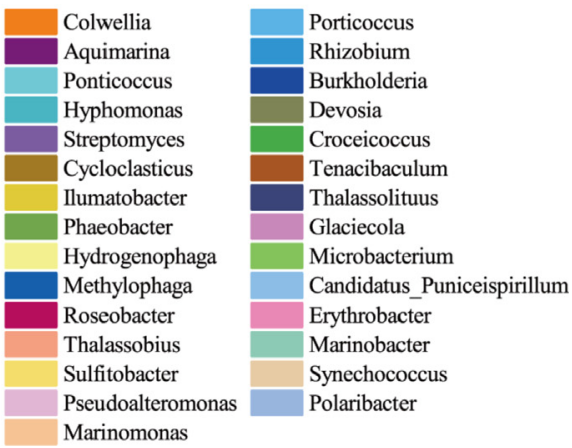

Fig. 2. Percent of community abundance in coastal waters on phylum (a) and genus (b) levels. Symbol -W refers to samples collected in winter. 
$22.45 \%$ of total bacterial genera of coastal water with a mean percentage of 3.12\% and samples collected from Bohai Area possessed more Synechococcus than those collected from the other areas. Candidatus_Pelagibacter averagely accounted for $2.92 \%$ of total bacterial genera with the highest percentage of $13.65 \%$ (B5T). Percentages of Candidatus_Pelagibacter in total bacterial genera of water samples collected from South China Sea Area were higher than those of water samples collected from the other areas. Nautella, Pseudomonas, Pseudoalteromonas, Marinobacterium, Planctomyces, and NS5 marine group also accounted for more than $1 \%$ of total bacterial genera on average. Some genera accounted for $9 \%-$ $30 \%$ of total bacterial genera in several water samples.
Neptuniibacter accounted for $20.10 \%$ of bacterial genera in E3 and Algoriphagus covered $29.94 \%$ of total genera in B6. Percentage of Rhodococcus and Malikia in total genera of Y1 reached $14.25 \%$ and $9.36 \%$, respectively. These results showed that bacterial communities at genus level might be influenced by multiple factors.

\subsection{Relationship among ARGs, intI1, 16S rRNA gene, and bacterial community in coastal waters}

Pearson correlation analysis was used to explore the possible relationship among water quality parameters, antibiotics, ARGs, and major bacteria in coastal waters (Fig. 3). OTC was significantly

(a)

(b)
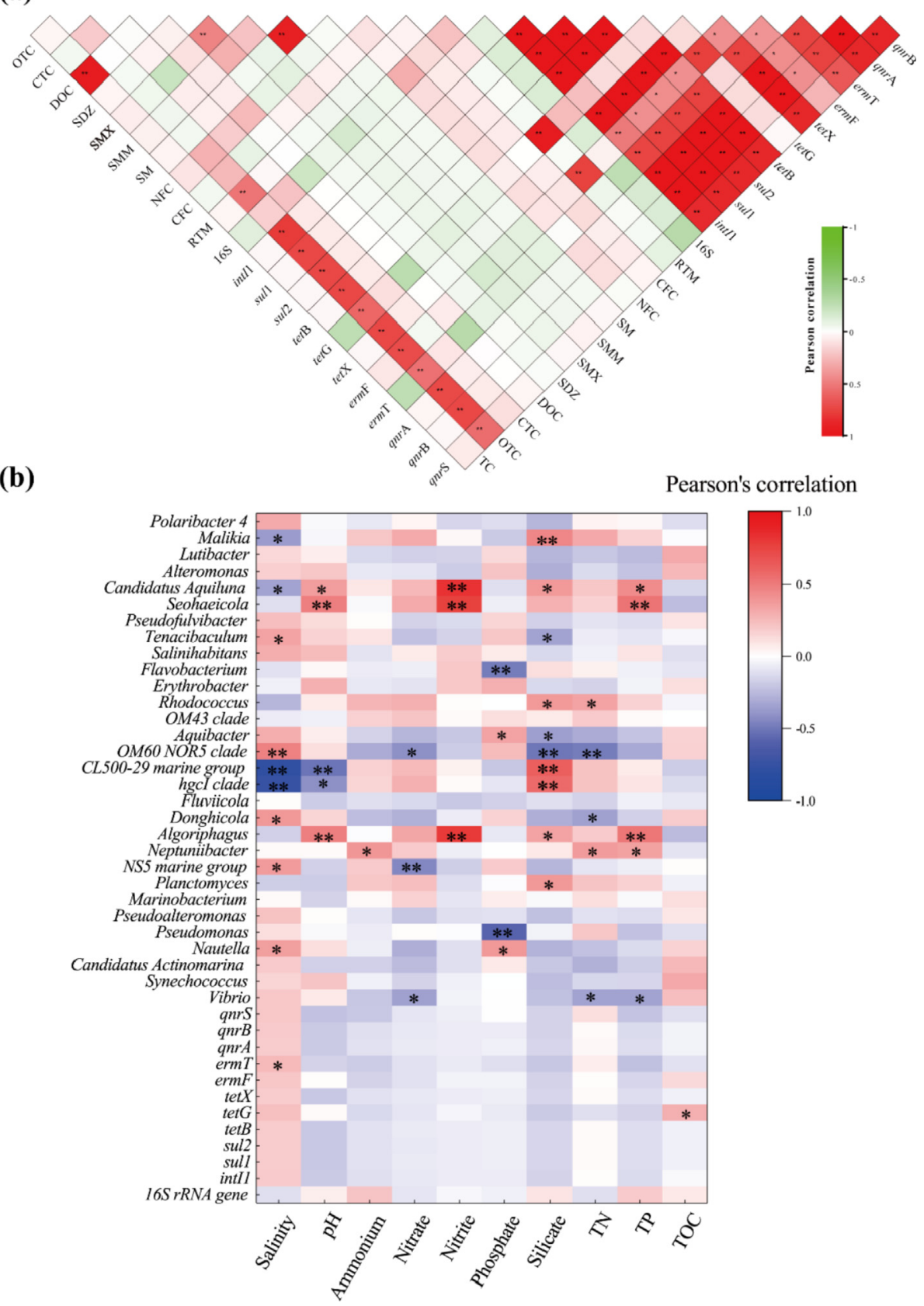

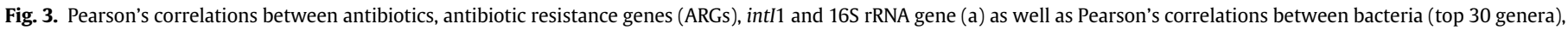

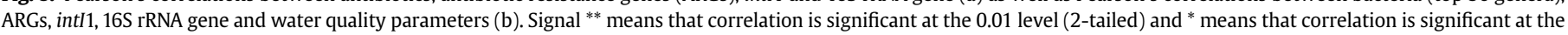
0.05 level (2-tailed). 
positively related to intI 1 and all target ARGs at $p<0.01$ and RTM was positively related to tetG and ermF at $p<0.01$ (Fig. 3a). The remaining target antibiotics were not related to target ARGs, illustrating that ARGs in coastal waters were influenced by complicated factors. Interestingly, tet $\mathrm{G}$ was only positively related to ermF that was only not related to qrnS (Fig. 3a). Significant positive relationship existed bewteen different ARGs and intI1. Salinity was significantly positively related to ermT at significance level of $p<0.05$ and TOC was positively related to tetG at $p<0.05$ (Fig. 3b). The remaining water quality parameters did not show significant relationship with 16S rRNA gene, intI1, and ARGs.

Bacteria in coastal water showed different patterns of correlating with water quality parameters (Fig. 3b). Salinity was significantly positively related to 5 bacteria while it was significantly negatively related to 4 bacteria. Relationship among silicate and some bacteria showed the opposite pattern of that among salinity and bacteria (Fig. 3b). Nitrate was negatively related to 3 bacteria while nitrite was positively related to 3 bacteria. TN was related to 5 bacteria while TOC was not related to the analyzed bacteria. Phosphorous was positively related to 2 bacteria at $p<0.05$ and negatively related to 2 bacteria at $p<0.01$ while TP was positively related to 4 bacteria and only negatively related to Vibrio.

Potential relationship among different samples in summer and winter was explored by PCA analysis (Fig. 4). Total variance of all samples, samples collected in summer, and samples collected in winter could be explained by two main components (Fig. 4a, c, and e). Interestingly, all samples collected in summer/winter could be devided two groups including group I (Y3T and Y3W) as well as group II (the remaining samples), illustrating that tail water and effluent of WWTPs were significantly different with the regular samples. However, B5T did not show significant difference with other regular coastal water samples (Fig. 4b, d, and f), which might be caused by its similar concentrations of antibiotics and water parameters with those of other samples.

Relationship among ARGs, intI1, 16S rRNA gene, and bacterial community were evaluated by network analysis (Fig. 5). Rhodobacter was the most active bacterium to be positively related to 12 bacteria and negatively related to 16 bacteria such as Mesoflavibacter, Rhodobium, Photobacterium (Fig. 5). Tenacibaculum was negatively related to 7 bacteria and positively related to 15 bacteria such as Pseudofulvibacter, Formosa, and Rhodobium. Positive or negative relationship simultaneously existed among the different bacteria of the coastal waters, showing that the complicated factors might have effects on the microbial community of the coastal waters in China. Most of ARGs did not show close relationship with bacteria in the water samples. MWH-UniP1 aquatic group and (a)

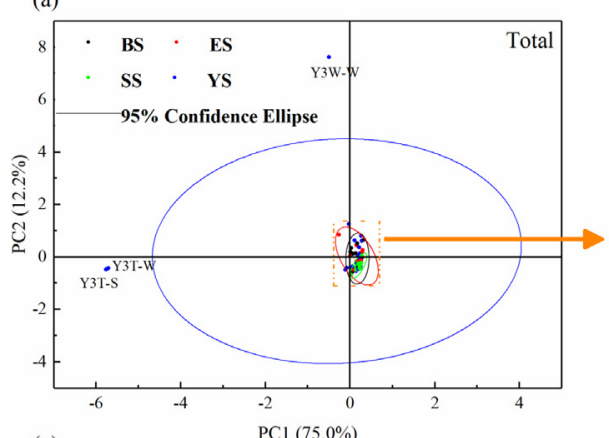

(c)
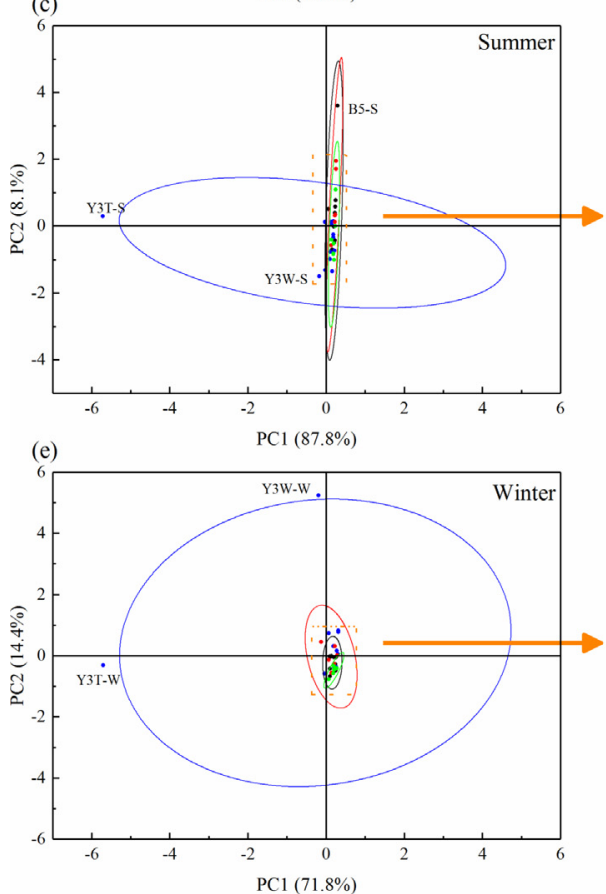

(b)

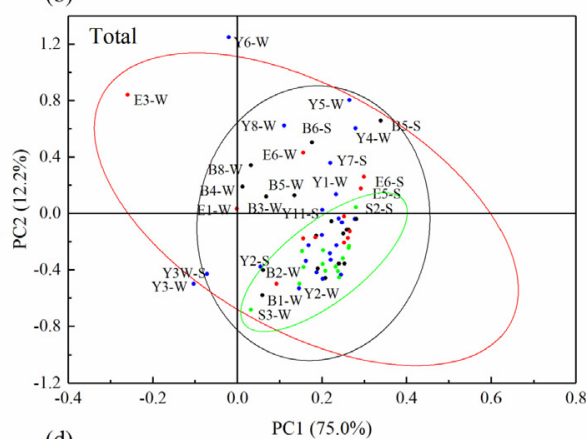

(d)

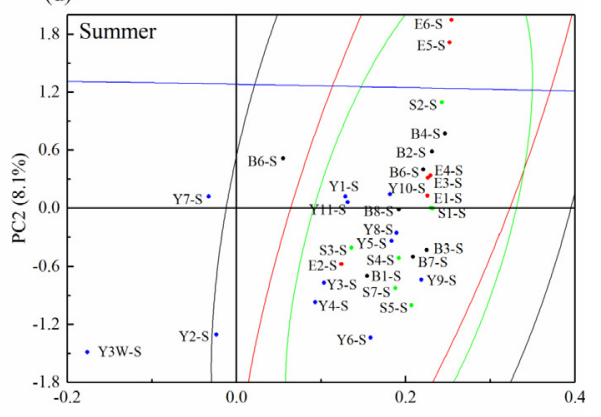

(f)

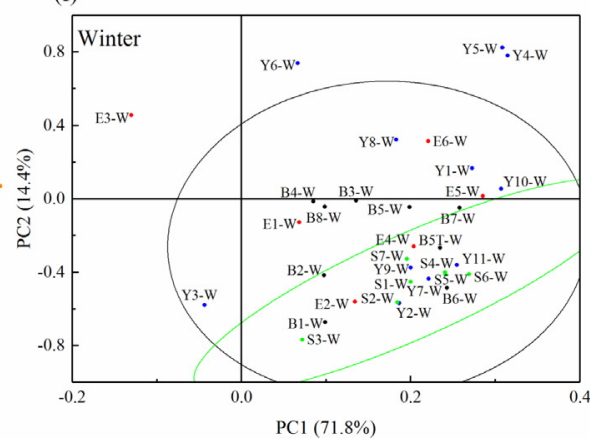

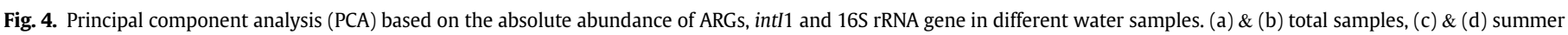
samples, (e) \& (f) winter samples. Figures (b), (d) and (f) were the enlarged figures of (a), (c) and (e), respectively. 


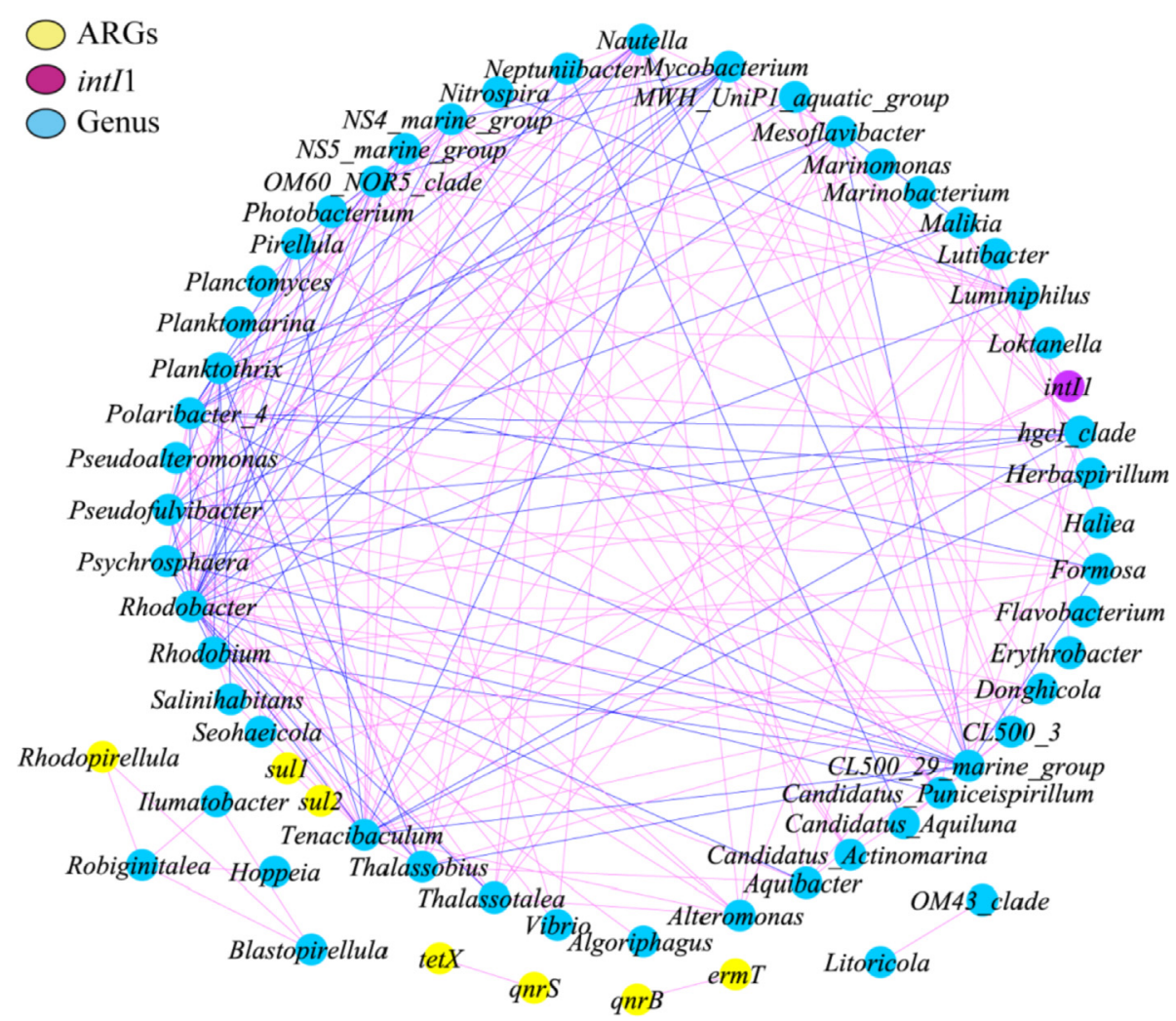

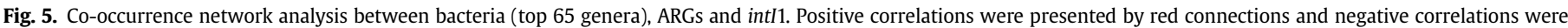
presented by blue connections (Spearman's correlation coefficient $|\rho|>0.6, p<0.05$ ).

Malikia were positively related to intil1 that was an important indicator for transfer of ARGs. However, the potential multi-drug bacteria were not detected based on the network analysis, indicating the relatively low horizontal gene transfer (HGT) of these detected ARGs among different bacteria in coastal water.

\section{Discussion}

Abundances of 16S rRNA gene, intI1, and ARGs in different coastal waters were compared (Fig. 6). B5T, Y3T, and Y3W were not included in Fig. 6 since they were tail water samples or effluent of WWTP. In general, the absolute abundances of 16S rRNA gene in coastal waters along coastline of China in this study were consistent with those in water of the Laizhou Bay (Li et al., 2018), but lower than 16S rRNA gene abundance in water of Duluth-Superior Harbor (LaPara et al., 2011) and water in two coastal areas (Geoje and Wando) of Korea (Germond and Kim, 2015). The absolute abundances of intI 1 in coastal waters of this study were consistent with those in water of Duluth-Superior Harbor (LaPara et al., 2011). Absolute abundance of total target ARGs in B3/B8, Y5/Y2, E3/E3, and S3/S7 was the maximal abundance of ARGs in coastal water samples collected in summer/winter from Bohai Area, Yellow Sea Area, East China Sea area, and South China Sea Area, respectively. The abundance of ARGs in water from Pear River Estuary (Chen et al., 2013) was lower than that of B8 in winter, but higher than that of the remaining water samples (Fig. 6a). Interestingly, the absolute abundances of ARGs in coastal water of China (this study) were much lower than those in water of Geoje. Moreover, the absolute abundances of ARGs in water of Wando were comparable with those in coastal waters of this study.

Relative abundance of total target ARGs in B3/B8, Y6/Y2, E2/E3, and $\mathrm{S} 3 / \mathrm{S} 2$ was the maximal relative abundance of ARGs in coastal

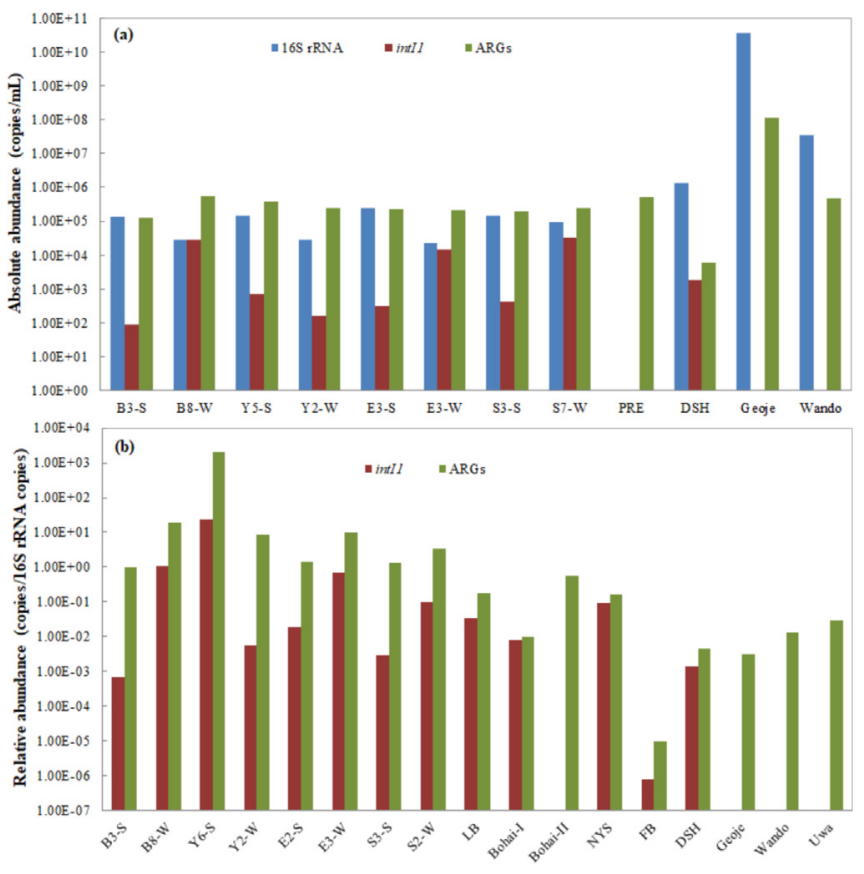

Fig. 6. Absolute abundances of ARGs, $16 \mathrm{~S}$ rRNA gene, and intI1 in coastal waters from China and other regions in the world (a) as well as relative abundances of ARGs and intI1 in coastal waters from China and other regions in the world (b). PRE is Pearl River Estuary; LB is Laizhou Bay; NYS is Noth Yellow Sea; FB is Frobisher Bay; DSH is DuluthSuperior Harbor. Symbols -W and -S refer to samples collected in winter and summer, respectively.

water samples collected in summer/winter from Bohai Area, Yellow Sea Area, East China Sea area, and South China Sea Area, 
respectively. Relative abundance of intI 1 in water of Bohai area previously reported (Niu et al., 2016) was higher than that in water of Bohai Area in summer of this study, but much lower than that in winter (Fig. 6b). Relative abundance of intI1 in coastal water of North Yellow Sea (Na et al., 2014) along coastline of China was higher than that in Y2 in winter, but much lower than that of Y6 in summer (Fig. 6b). Relative abundances of intI 1 in coastal water of Frobisher Bay (Neudorf et al., 2017) and Duluth-Superior Harbor (LaPara et al., 2011) were lower than those in this study, illustrating higher horizontal transfer risks of ARGs in coastal waters along coastline of China. Relative abundances of ARGs in Bohai Area previously reported (Niu et al., 2016; Zhang et al., 2018) were lower than those in the same area of this study. Relative abundances of ARGs in Noth Yellow Sea previously reported (Na et al., 2014) were lower than those in the similar area of this study. Relative abundances of ARGs in waters of Frobisher Bay, Duluth-Superior Harbor, Geoje, Wando, and the Uwa Sea (Germond and Kim, 2015; LaPara et al., 2011; Neudorf et al., 2017; Suzuki et al., 2019) were also much lower than those in this study (Fig. 6b).

Absolute abundance of $16 \mathrm{~S}$ rRNA gene in aquaculture tail water (B5T and Y3T) in both summer and winter was consistent with that in water of mariculture farm previous reported (Wang et al., 2019) while absolute abundance of 16S rRNA gene in the effluent of WWTP in summer was significantly lower than that in water of mariculture farm. Absolute abundance of intI1 in B5T was significantly lower than that in water of mariculture farm while absolute abundance of intI1 in Y3T was higher than that in fish pond water of mariculture farm (Wang et al., 2019). Absolute abundance of ARGs in B5T, Y3T, and Y3W was lower than that in the recycled water of the mariculture farm (Wang et al., 2019) while relative abundance of ARGs in B5T, Y3T, and Y3W was 1-6 orders of magnitude higher than that in aquaculture area of Japan (Suzuki et al., 2019) or effluent of coastal aquaculture of Korea (Jang et al., 2018). Relatively high abundances of ARGs in aquaculture tail water and effluent of WWTPs illustrated that tail water and WWTP effluents should be important sources of ARGs in coastal waters.

\section{Conclusions}

This study provided comprehensive information on distribution of ARGs in coastal waters of China at a continental scale. The absolute abundances of $16 \mathrm{~S}$ rRNA gene, intI1, and target ARGs in coastal waters along the $18000 \mathrm{~km}$ coastline of China showed drastic spatio-temporal variation at a continental scale. The average total absolute abundance of ARGs in coastal waters reached the highest in Bohai Area during winter and in East China Sea Area during summer. Quinolone resistance genes were the dominant ARGs in coastal water. Bacterial communities of different water samples showed significant difference both at phylum and genus levels. Significant positive relationship existed between different ARGs and intI1. Most of ARGs did not show close relationship with bacteria in the coastal water samples based on network analysis. The abundances of target ARGs in coastal waters of China were comparable with those in the other regions of China and other countries. These findings indicate that ARGs pollution has become a crucial stress affecting the sustainable development of coastal regions. It is urgent to take effective and efficient measures to control ARGs pollution in coastal regions.

\section{Acknowledgements}

This work was supported by National Natural Science Foundation of China (41877131), Taishan Scholar Program of Shandong Province (No. tsqn201812116), One Hundred Talents Program of Chinese Academy of Sciences (Y629041021), and Two-Hundred
Talents Plan of Yantai (Y739011021). The authors would like to thank the editor and reviewers for their valuable suggestions and comments on this manuscript.

\section{References}

Bondarczuk, K., Markowicz, A., Piotrowska-Seget, Z., 2016. The urgent need for risk assessment on the antibiotic resistance spread via sewage sludge land application. Environ. Int. 87, 49-55.

Calero-Caceres, W., Muniesa, M., 2016. Persistence of naturally occurring antibiotic resistance genes in the bacteria and bacteriophage fractions of wastewater. Water Res. 95, 11-18.

Chen, B., He, R., Yuan, K., Chen, E., Lin, L., Chen, X., Sha, S., Zhong, J., Lin, L., Yang, L., Yang, Y., Wang, X., Zou, S., Luan, T., 2017. Polycyclic aromatic hydrocarbons (PAHs) enriching antibiotic resistance genes (ARGs) in the soils. Environ. Pollut. 220, 1005-1013.

Chen, B., Liang, X., Huang, X., Zhang, T., Li, X., 2013. Differentiating anthropogenic impacts on ARGs in the Pearl River Estuary by using suitable gene indicators. Water Res. 47, 2811-2820.

Gao, Q., Li, Y., Qi, Z., Yue, Y., Min, M., Peng, S., Shi, Z., Yang Gao, Y., 2018. Diverse and abundant antibiotic resistance genes from mariculture sites of China's coastline. Sci. Total Environ. 630, 117-125.

Germond, A., Kim, S.-J., 2015. Genetic diversity of oxytetracycline-resistant bacteria and tet(M) genes in two major coastal areas of South Korea. J. Global Antimicrob. Resist. 3, 166-173.

Huang, L., Xu, Y.-B., Xu, J.-X., Ling, J.-Y., Chen, J.-L., Zhou, J.-L., Zheng, L., Du, Q.-P., 2017. Antibiotic resistance genes (ARGs) in duck and fish production ponds with integrated or non-integrated mode. Chemosphere 168, 1107-1114.

Jang, H.M., Kim, Y.B., Choi, S., Lee, Y., Shin, S.G., Unno, T., Kim, Y.M., 2018. Prevalence of antibiotic resistance genes from effluent of coastal aquaculture, South Korea. Environ. Pollut. 233, 1049-1057.

Jones, C.E., Maddox, A., Hurley, D., Barkovskii, A.L., 2018. Persistence of bacterial pathogens, antibiotic resistance genes, and enterococci in tidal creek tributaries. Environ. Pollut. 240, 875-883.

LaPara, T.M., Burch, T.R., McNamara, P.J., Tan, D.T., Yan, M., Eichmiller, J.J., 2011. Tertiary-treated municipal wastewater is a significant point source of antibiotic resistance genes into duluth-superior harbor. Environ. Sci. Technol. 45, 9543-9549.

Larrañaga, O., Brown-Jaque, M., Quirós, P., Gómez-Gómez, C., Blanch, A.R., Rodríguez-Rubio, L., Muniesa, M., 2018. Phage particles harboring antibiotic resistance genes in fresh-cut vegetables and agricultural soil. Environ. Int. 115, 133-141.

Li, Q., Na, G., Zhang, L., Lu, Z., Gao, H., Li, R., Jin, S., 2018. Effects of corresponding and non-corresponding contaminants on the fate of sulfonamide and quinolone resistance genes in the Laizhou Bay, China. Mar. Pollut. Bull. 128, 475-482.

Lin, L., Yuan, K., Liang, X., Chen, X., Zhao, Z., Yang, Y., Zou, S., Luan, T., Chen, B., 2015 Occurrences and distribution of sulfonamide and tetracycline resistance genes in the Yangtze River Estuary and nearby coastal area. Mar. Pollut. Bull. 100, 304-310.

Lu, J., Wu, J., Zhang, C., Zhang, Y., Lin, Y., Luo, Y., 2018. Occurrence, distribution, and ecological-health risks of selected antibiotics in coastal waters along the coastline of China. Sci. Total Environ. 644, 1469-1476.

Lu, J., Zhang, C., Wu, J., Lin, Y., Zhang, Y., Yu, X., Zhang, Z., 2019a. Pollution, sources, and ecological-health risks of polycyclic aromatic hydrocarbons in coastal waters along coastline of China. Hum. Ecol. Risk Assess. https://doi.org/10.1080/ 10807039.2018.1548899 (in press).

Lu, J., Zhang, Y., Wu, J., Wang, J., Zhang, C., Lin, Y., 2019b. Occurrence and spatial distribution of antibiotic resistance genes in the Bohai Sea and Yellow Sea areas, China. Environ. Pollut. 252, 450-460.

Lu, J., Zhang, Y., Wu, J., Luo, Y., 2019c. Effects of microplastics on distribution of antibiotic resistance genes in recirculating aquaculture system. Ecotoxicol. Environ. Saf. 184, 109631

Lv, B., Cui, Y., Tian, W., Li, J. Xie, B., Fang Yin, F., 2018. Abundances and profiles of antibiotic resistance genes as well as co-occurrences with human bacterial pathogens in ship ballast tank sediments from a shipyard in Jiangsu Province, China. Ecotoxicol. Environ. Saf. 157, 169-175.

Marti, E., Huerta, B., Rodríguez-Mozaz, S., Barcel, D., Marce, R., Balcazar, J.L., 2018. Abundance of antibiotic resistance genes and bacterial community composition in wild freshwater fish species. Chemosphere 196, 115-119.

McCann, C.M., Christgen, B., Roberts, J.A., Su, J.-Q., Arnold, K.E., Gray, N.D., Zhu, Y.-G., Graham, D.W., 2019. Understanding drivers of antibiotic resistance genes in High Arctic soil ecosystems. Environ. Int. 125, 497-504.

Na, G., Zhang, W., Zhou, S., Gao, H., Lu, Z., Wu, X., Li, R., Oiu, L., Cai, Y., Yao, Z., 2014. Sulfonamide antibiotics in the Northern Yellow Sea are related to resistant bacteria: implications for antibiotic resistance genes. Mar. Pollut. Bull. 84, 70-75.

Neudorf, K.D., Huang, Y.N., Ragush, C.M., Yost, C.K., Jamieson, R.C., Hansen, L.T., 2017. Antibiotic resistance genes in municipal wastewater treatment systems and receiving waters in Arctic Canada. Sci. Total Environ. 598, 1085-1094.

Niu, Z.G., Zhang, K., Zhang, Y., 2016. Occurrence and distribution of antibiotic resistance genes in the coastal area of the Bohai Bay, China. Mar. Pollut. Bull. 107, 245-250.

Olanrewaju, T.O., McCarron, M., Dooley, J.S.G., Arnscheidt, J., 2019. Transfer of 
antibiotic resistance genes between Enterococcus faecalis strains in filter feeding zooplankton Daphnia magna and Daphnia pulex. Sci. Total Environ. 659, 1168-1175.

Pan, K., Wang, W.-X., 2012. Trace metal contamination in estuarine and coastal environments in China. Sci. Total Environ. 421-422, 3-16.

Qiu, Y., Zhang, J., Li, B., Wen, X., Liang, P., Huang, X., 2018. A novel microfluidic system enables visualization and analysis of antibiotic resistance gene transfer to activated sludge bacteria in biofilm. Sci. Total Environ. 642, 582-590.

Su, H.-C., Pan, C.-G., Ying, G.-G., Zhao, J.-L., Zhou, L.-J., Liu, Y.-S., Tao, R., Zhang, R.-O., He, L.-Y., 2014. Contamination profiles of antibiotic resistance genes in the sediments at a catchment scale. Sci. Total Environ. 490, 708-714.

Suzuki, S., Nakanishi, S., Tamminen, M., Yokokawa, T., Sato-Takabe, Y., Ohta, K. Chou, H.-Y., Muziasari, W.I., Virta, M., 2019. Occurrence of sul and tet(M) genes in bacterial community in Japanese marine aquaculture environment throughout the year: profile comparison with Taiwanese and Finnish aquaculture waters. Sci. Total Environ. 669, 649-656.
Wang, J.-H., Lu, J., Wu, J., Zhang, Y., Zhang, C., 2019. Proliferation of antibiotic resistance genes in coastal recirculating mariculture system. Environ. Pollut. 248, 462-470.

Wang, J.-H., Lu, J., Zhang, Y.-X., Wu, J., Luo, Y., Liu, H., 2018. Metagenomic analysis of antibiotic resistance genes in coastal industrial mariculture systems. Bioresour. Technol. 253, 235-243.

Xu, Y., Xu, J., Mao, D., Luo, Y., 2017. Effect of the selective pressure of sub-lethal leve of heavy metals on the fate and distribution of ARGs in the catchment scale. Environ. Pollut. 220, 900-908.

Yang, Y., Zhou, R., Chen, B., Zhang, T., Hu, L., Zou, S., 2018. Characterization of airborne antibiotic resistance genes from typical bioaerosol emission sources in the urban environment using metagenomic approach. Chemosphere 213 463-471.

Zhang, Y., Niu, Z., Zhang, Y., Zhang, K., 2018. Occurrence of intracellular and extracellular antibiotic resistance genes in coastal areas of Bohai Bay (China) and the factors affecting them. Environ. Pollut. 236, 126-136. 